\section{Cahiers de Narratologie}

Analyse et théorie narratives

10.2 | 2001

La voix narrative

\title{
La voix narrative démultipliée : intertextualité classique et ironie dans les écrits proustiens
}

\section{Sylvaine Landes-Ferrali}

\section{(2) OpenEdition}

1 Journals

Édition électronique

URL : http://journals.openedition.org/narratologie/10197

DOI : 10.4000/narratologie.10197

ISSN : 1765-307X

Éditeur

LIRCES

\section{Édition imprimée}

Date de publication : 1 janvier 2001

Pagination : 130-141

ISBN : 2914561032

ISSN : 0993-8516

\section{Référence électronique}

Sylvaine Landes-Ferrali, « La voix narrative démultipliée : intertextualité classique et ironie dans les écrits proustiens », Cahiers de Narratologie [En ligne], 10.2 | 2001, mis en ligne le 01 janvier 2001, consulté le 11 juin 2020. URL : http://journals.openedition.org/narratologie/10197 ; DOI : https:// doi.org/10.4000/narratologie.10197 


\title{
LA VOIX NARRATIVE DÉMULTIPLIÉE : INTERTEXTUALITÉ CLASSIQUE ET IRONIE DANS LES ÉCRITS PROUSTIENS
}

\author{
Sylvaine LANDES -FERRALI \\ Université d'Aix-Marseille
}

Dans une lettre adressée à René Blum, dans laquelle il évoque son roman, Proust fait remarquer : "C'est encore du roman que cela s'écarte le moins; il y a un monsieur qui raconte et qui dit: $\mathrm{Je}^{1}$ ». La même année, dans le texte répertorié dans Contre Sainte-Beuve ${ }^{2}$ sous le titre Swann expliqué par Proust, qui correspond à une entrevue accordée au journal Le Temps, on peut lire : «(...) dans ce premier volume, vous verrez le personnage qui raconte, qui dit : «je » (et qui n'est pas moi) » etc. Une lettre de 1920 apporte une précision supplémentaire, un peu contradictoire, mais précieuse, en faisant allusion « au personnage qui dit « je » (et qui n'est pas toujours moi) ${ }^{3}$ ».

La première mention situe simplement l'œuvre dans une perspective romanesque particulière, qui pourrait l'apparenter à la forme autobiographique, la seconde la réfute totalement, et à juste titre, replaçant le roman dans sa qualité intrinsèque, la troisième enfin, et c'est celle qui nous intéresse, diversifie le domaine de l'énonciation, qui au regard de l'intertextualité ${ }^{4}$

${ }^{1}$ Correspondance de Marcel Proust, édition Philip Kolb, Paris, Plon, 1970-1993, tome XII, p. 92. Nous y ferons référence de façon abrégée, Corr., t. I, II, III, etc.

${ }^{2}$ Contre Sainte-Beuve, précédé de Pastiches et mélanges, et suivi de Essais et articles, Gallimard, 1971, Bibl. de la Pléiade, p. 557.

${ }^{3}$ Corr., tome XIX, p. 78.

4 Nous entendrons par intertextualité la mise en relation par coprésence d'un texte littéraire d'emprunt avec un texte d'accueil, quelle qu'en soit la modalité, que la référence s'inscrive in absentia 
nous conduira à nous interroger d'une part sur les rapports entretenus entre le narrateur et le héros dans le cadre du récit rétrospectif, et particulièrement par rapport à une écriture antérieure, d'autre part sur les liens qui peuvent unir le narrateur et le romancier, qui de son propre aveu, s'interpose quelquefois dans son discours.

Récit rétrospectif, la Recherche pose déjà une dissociation : la voix vieillissante du narrateur met en scène le jeune homme qu'il fut et donne à entendre la voix qui fut la sienne. Il y a d'emblée disjonction, mais il en est d'autres : Marcel Muller ${ }^{5}$ ne distingue pas moins de sept voix différentes dans le roman. Il fait remarquer que la question des rapports entre le héros-narrateur et la personne de Proust est extrêmement compliquée ; toutefois, faisant la part de « l'Auteur (celui qui avoue la présence de son moi créateur dans le roman) » et celle de "l'Homme (celui qui avoue la présence de son moi quotidien) " il ne manque pas de préciser : "Cet aveu est peut-être mensonger ", et il est évident que cela est à prendre en compte de façon permanente.

La pratique de la citation classique ajoute une dimension supplémentaire, et la voix narrative dans ce cas s'enrichit d'un élément nouveau, car la dualité du personnage-narrateur et de l'auteur peut s'estomper au profit d'une plus large part accordée à l'auteur.

L'usage qui est fait de l'intertextualité classique rappelle alors les jeux entretenus autour du quotidien de l'écriture privée, dans la correspondance, et pose des interrogations sur l'intrusion d'un discours qui doit beaucoup à un héritage culturel et affectif.

(par allusion, commentaire ou paraphrase), ou in praesentia (par citation).

5 Les problèmes posés par l'identité du je dans la Recherche ne sont pas nouveaux. Après Jean Pouillon, "Les règles du Je ", in Les Temps modernes, XII (1956-1957), voir Marcel Muller, Les voix narratives dans « La Recherche du temps perdu ", Droz, Genève, 1983. J.-Yves Tadié, entre temps, dans Proust et le roman, Gallimard, 1971, coll. Tel, et sur un plan différent, avait posé les problèmes du narrateur dans son chapitre du même titre, pp. 17-33. Nous placerons notre réflexion dans le cadre exclusif de la relation à l'intertextualité classique. 
Triple superposition des voix : celle inhérente au statut du récit, celle du passé personnel de l'écrivain, celle enfin directement impliquée par le texte emprunté. L'ironie engendrée alors par la rencontre de deux types de discours souvent en position de décalage, outre qu'elle confère au texte sa portée comique, consacre et enrichit, en vertu de la parole dédoublée qu'elle sous-tend, certains aspects polyphoniques du texte proustien.

Si le narrateur de la Recherche en tant que personnage ne cite qu'assez peu sur le mode direct les textes classiques, en tant que conducteur du récit en revanche, il a la haute main sur les innombrables rapports établis avec la littérature du XVII ${ }^{e}$ siècle. Celle-ci tient dans la correspondance de l'écrivain une très large place, et à observer les textes cités, leur mode de citation, et le ton qu'ils confèrent à bon nombre des lettres, on constate que l'œuvre de fiction accorde si l'on peut dire, un droit d'entrée fréquent à son auteur. Dans une lettre écrite en $1918^{6}$ à son ami et banquier Lionel Hauser, Proust se plaint de ses peines de cœur ${ }^{7}$, et il ajoute en guise de commentaire : "Il est triste a dit La Bruyère d'aimer sans une grande fortune ${ }^{8} »$. La parole du moraliste s'interpose dans le fil du discours de l'épistolier, et les deux voix demeurent en parallèle dans le texte. C'est de façon rigoureusement identique que le narrateur de la Recherche ${ }^{9}$ introduit le texte de La Bruyère, dans A l'ombre des jeunes filles en fleurs ${ }^{10}$, lorsque, après avoir vendu une potiche de Chine pour faire

${ }^{6}$ Corr., tome XVII, p. 367.

${ }^{7}$ Il s'agit probablement de celles occasionnées par Henri Rochat, employé au Ritz, qui deviendra le secrétaire de l'écrivain ; voir Corr., tome XVII, pp. VIII-IX.

8 «Il est triste d'aimer sans une grande fortune, et qui nous donne les moyens de combler ce que l'on aime, et le rendre si heureux qu'il n'ait plus de souhaits à faire ", La Bruyère, Les Caractères, "Du cœur ", fragment 20.

${ }^{9}$ Nous ferons référence à l'édition de $A$ la recherche du temps perdu de J.-Yves Tadié et alii, 4 vol., Gallimard, Bibl. de la Pléiade, 1987-1989, de façon abrégée, RTP., I, II, III, IV.

${ }^{10}$ RTP., III, p. 613. 
des cadeaux à Gilberte il l'aperçoit en compagnie d'un jeune homme et qu'il conclut à une impossibilité du bonheur.

Mais si je n'avais pas fait cet arrêt (...) je n'eusse pas rencontré Gilberte et ce jeune homme. Ainsi un même fait porte des rameaux opposites et le malheur qu'il engendre annule le bonheur qu'il avait causé. Il m'était arrivé le contraire de ce qui se produit si fréquemment. On désire une joie, et le moyen matériel de l'atteindre fait défaut. «Il est triste, a dit La Bruyère, d'aimer sans une grande fortune. »

On retrouve ainsi la parole dédoublée de l'épistolier dans celle du narrateur, dans un discours emprunté commun, qui sert de commentaire et de complément à son propre discours. Une forme de dialogisme ${ }^{11}$ s'instaure au cœur du texte de fiction mais il se multiplie des communications qui s'établissent entre les différentes voix, celle du narrateur du roman, celle de l'auteur tel qu'il apparaît dans la correspondance et dont on a l'écho dans la fiction. Le ton se maintient ici sur le mode grave, du moins sérieux, même si l'on peut déceler dans la confrontation du texte du moraliste classique avec un quotidien trivial et ambigu (les amours avec un employé d'hôtel), et celui minime et anodin (les amours enfantines), une discordance ${ }^{12}$ qui engendre une voix supplémentaire.

Elle prête en ce cas aux voix narratives un ton que la citation classique va enrichir de la parodie ${ }^{13}$ et du travestissement. Ceux-ci sont prépondérants dans la correspondance de l'écrivain qui mêle joyeusement la parole des grands

11 Au sens où se confrontent dans le discours des voix hétérogènes, sans que l'on sorte de la littérature, ni qu'il soit question d'enracinement social, comme l'entend Bakhtine dans sa théorie.

12 Celle-ci va de pair avec le burlesque défini par Ch. Perrault dans son IIIe Parallèle des anciens et des modernes, comme " une espèce de ridicule (qui) consiste dans la disconvenance de l'idée qu'on donne d'une chose avec son idée véritable", cité par A. Viala, article «Burlesque » dans le Dictionnaire des Littératures de langue française, J.-P. de Beaumarchais, D. Couty, A. Rey, éd. Bordas, 1987, tome I, p. 352 .

13 Selon la définition qu'en donne Dumarsais, repris par Genette (Palimpsestes, Seuil, 1983, p. 23) : "Procédé où l'on détourne dans un sens railleur des vers qu'un autre a fait dans une vue différente. " 
classiques à la sienne propre dans un entrelacs de discours qui rend à l'intertextualité son sens premier. Les exemples sont innombrables, ils prennent essentiellement appui sur les noms les plus connus du XVII siècle $^{14}$, et servent indifféremment l'écriture privée et l'écriture littéraire. Dans la correspondance, le mode d'insertion de la citation permet souvent d'oublier la voix du scripteur et de lui attribuer grâce au texte d'emprunt et à son contexte, une voix seconde, qui crée le plus souvent des effets comiques. Proust, dans une lettre à Montesquiou en 1910, après avoir exprimé de regrets de n'avoir pu le rencontrer, ajoute : «Quel bonheur ce sera pour moi si je peux vous voir et de satisfaire cette "grande faim (de vous revoir et de vous entendre) qu'à vos yeux on $\operatorname{expose}^{15 "}$ ". Il y a ici une allusion à Molière, et particulièrement aux vers prononcés par Trissotin lorsqu'il s'apprête à lire son sonnet: " Pour cette grande faim qu'à mes yeux on expose / Un plat seul de huit vers me semble peu de chose ${ }^{16}$ ».

L'énonciation qui entre en jeu de prime abord dans la lettre de Proust laisse croire qu'il parle au nom de Trissotin qui se prépare à faire entendre son sonnet à ses admiratrices, Philaminte, Armande, et Bélise, qui ont manifesté leur impatience d'entendre le sonnet. Or, par la transposition des pronoms possessifs (vos yeux pour mes yeux), l'épistolier délègue le rôle de Trissotin à son destinataire, et s'octroie celui du public impatient, non pas de l'entendre mais, compte tenu de l'adaptation du texte de Molière à sa situation, de le revoir. Ce jeu est fréquent dans la correspondance, étroitement lié à la transposition parodique et au détournement à

14 La Fontaine permet à Proust de plaisanter avec son ami Lucien Daudet (Corr. tome IX, p. 99), par la transformation du il en je, et l'usurpation implicite du nom du héros de la fable : «Bien que j'aie fait je le vois deux parts de ma vie l'une à vous écrire et "l'autre à ne rien faire », je ne peux pas répondre à votre lettre. " L'épistolier fait ici référence à l' "Epitaphe d'un paresseux »: "Jean s'en alla comme il était venu, / Mangea le fonds avec le revenu, / Tint les trésors chose peu nécessaire. Quant à son temps bien le sut dispenser : / Deux parts en fit, dont il soulait passer / L'une à dormir, et l'autre à ne rien faire. " (Euvres diverses, Gallimard, 1958, Bibl. de la Pléiade, p. 495-496).

${ }^{15}$ Corr., tome X, p. 51.

${ }^{16}$ Les Femmes savantes, acte III, scène II, vers 747. 
d'autres fins du discours qu'il implique. Il induit une prise de parole seconde qu'il superpose si l'on peut dire sur l'initiale, créant double langage et par là même une forme ambiguë de relation à son propre discours. Cette ambiguïté revêt un caractère particulier dans le cadre de l'intertextualité racinienne.

Un exemple mérite qu'on s'y arrête, pour ce qu'il permet d'élargir le champ d'interprétation, par la reprise de la référence en différents lieux d'écriture, et de considérer les effets dans le texte de la fusion du moi créateur et du moi quotidien avec le narrateur et le personnage. Il s'agit de la référence à Esther.

Largement présente dans différents écrits de Proust, elle devient outil herméneutique : compromettant l'univocité, elle laisse entrevoir dans l'œuvre du romancier, derrière le masque du texte racinien, bien des aspects de ce que l'on pourrait appeler la problématique de l'homme. Nous nous limiterons à ce qui apparaît comme l'identification à Assuérus, qui s'exerce principalement à travers deux scènes dont quelques vers alimentent le texte proustien. Les uns sont prononcés dans la tragédie par Esther, au sujet des lois qui régissent les entrevues que l'on peut avoir avec les rois :

Hélas ! ignorez-vous quelles sévères lois / Aux timides mortels cachent ici les rois? Au fond de leur palais leur majesté terrible / Affecte à leurs sujets de se rendre invisible ; Et la mort est le prix de tout audacieux / Qui sans être appelé se présente à leurs yeux, Si le Roi dans l'instant, pour sauver le coupable / Ne lui donne à baiser son sceptre redoutable. / Rien ne met à l'abri de cet ordre fatal, / Ni le rang, ni le sexe, et le crime est égal. ${ }^{17}$

Les autres prononcés par Assuérus, concernent le moment où Esther entre chez le roi sans y être appelée, et ne pouvant dominer son trouble, tombe évanouie :

Sans mon ordre on porte ici ses pas ? / Quel mortel insolent vient chercher le trépas? / Gardes ... C'est vous, Esther ? Quoi ? sans être attendue ${ }^{18}$ ?

${ }^{17}$ Racine, Esther, Acte I, Scène III, vers 191-200.

18 Ibidem, Acte II, Scène VII, vers 630-632. 


\section{(...)}

Esther, que craignez-vous ? Suis-je pas votre frère ? / Est-ce pour vous qu'est fait un ordre si sévère ? / Vivez, le sceptre d'or, que vous tend cette main, / Pour vous de ma clémence est un gage certain. ${ }^{19}$

Dans Contre Sainte-Beuve ${ }^{20}$, l'épisode «Conversation avec Maman $^{21}$ », instaure un dialogue entre la mère et le fils, fondé sur l'interdiction de pénétrer dans la chambre du narrateur, qui prend appui sur ces vers raciniens. C'est par le biais de la citation qu'en fait le personnage de la mère que se réalise l'identification à Assuérus. Le narrateur en effet dit sa crainte que son frère Robert n'entre directement chez lui. La mère répond alors en utilisant, modifiés pour la circonstance, certains des vers que nous citions plus haut : "Peut-il donc ignorer quelle sévère loi / Aux timides mortels cachent ici notre roi, / Que la mort est le prix de tout audacieux / Qui sans être appelé se présente à ses yeux ? ». Les modifications apportées consacrent le narrateur, qu'il est difficile dans cet avant-texte de ne pas identifier à l'auteur ou au moins de ne pas l'en rapprocher étroitement (Robert est le prénom du frère de Proust), comme l'enfant roi, auquel la mère, sous le masque d'Esther, donne la réplique de façon ambiguë. La suite du texte confirme ces transpositions.

(...) J'appelle, aucun bruit ne répond. J'appelle de nouveau, j'entends des pas furtifs, une hésitation à ma porte qui grince.

- Maman.

- Tu m'avais bien appelé mon chéri ?

${ }^{19}$ Ibidem, vers 637-640.

20 Première ébauche de la Recherche, hormis Jean Santeuil, l'ouvrage est évoqué par Proust dans une lettre à Georges de Lauris en 1908 : «J'ai en quelque sorte deux articles bâtis en ma pensée (...) : l'un est un essai de forme classique (...) ; l'autre débuterait par le récit d'une matinée : maman viendrait près de mon lit, et je lui raconterais l'article que je veux faire sur Sainte-Beuve, et je le lui développerais " (cité dans Contre Sainte-Beuve, Bibl. de la Pléiade, op. cit., p. 822).

21 C'est le titre qui figure dans l'édition de Bernard de Fallois, Gallimard, 1954, NRF, coll. Idées, pp. 135-149; voir aussi Contre Sainte-Beuve, Bibl. de la Pléiade, op. cit., à la rubrique Projets de préface, pp. 217-218. 
- Oui

- Je te dirai que j'avais peur de m'être trompée et que mon Loup me dise :

Suivent les vers prononcés par Assuérus lorsqu'Esther dans la tragédie entre chez lui ; le narrateur, donnant la réplique, emploie cette fois-ci directement les vers qui consacrent l'éminence et l'exclusivité de la place occupée par Esther ${ }^{22}$. Le passage s'achève, du moins en ce qui concerne l'intertextualité racinienne, par le commentaire de la mère : «-Cela n'empêche pas que je crois que, si je l'avais réveillé, je ne sais pas si mon Loup m'aurait si béatement tendu son sceptre d'or. "

Le je du narrateur devenant celui d'Assuérus, dans l'ébauche de ce qui deviendra le dialogue avec Albertine, la mère-Esther préparant Albertine-Esther, laissent entrevoir toutes les combinaisons que l'on peut établir autour de la multiplication des voix et de l'intertextualité racinienne.

On ne peut que conclure au double je et au double jeu, lorsqu'on se penche sur la correspondance.

Le texte y est utilisé de façon lacunaire, à deux reprises. Proust écrivant à Montesquiou en 191723 indique qu'il a "avec Assuérus cette seule ressemblance qu'on n'entre pas dans (sa) chambre " "sans y être appelé" ». Il réitère la comparaison l'année suivante dans une lettre à Lionel Hauser, en l'adaptant et l'interprétant : « (...) pareil au roi Assuérus jamais on ne m'éveille ${ }^{24} »$.

22 Esther tient une place considérable dans les écrits proustiens, quel que soit le mode de traitement adopté ; voir Antoine Compagnon, "Proust sur Racine », Revue des Sciences humaines, tome LXVII, oct.déc. 1984, pp. 39-64. Le cadre étroit de cette étude ne nous permet pas de développer davantage la relation existant entre la tragédie racinienne et l'emploi qui en est fait dans l'œuvre. Rappelons que Mme Proust cite Esther dans les lettres adressées à son fils, et que l'écrivain dans Contre Sainte-Beuve (édition Bernard de Fallois) évoque l'amour particulier de sa mère pour cette pièce de Racine, en même temps que "les belles lignes de son visage juif ». La voix narrative que l'on entend dans la Recherche s'en trouve considérablement enrichie et diversifiée.

23 Corr., tome XVI, p. 245.

24 Ibidem, tome XVII, p. 278. 
La mise en relation de l'espace de la chambre, avec le palais du roi Assuérus, double celle que l'écrivain établit entre son sommeil et en l'occurrence son travail, puisqu'on sait que Proust écrit la nuit et qu'il n'est pas, durant le jour, souvent accessible. Faisant référence à un lieu et à un personnage dont il se saisit de la fonction et de la parole, et au-delà de la simple plaisanterie inhérente au travestissement et au burlesque ${ }^{25}$ la voix de l'épistolier déjà ici dédoublée, est à la jonction de celles du héros du roman et de l'auteur telles qu'elles se laissent entendre à travers la pratique intertextuelle. L'élément du sommeil, présent dans ce qui constitue l'ébauche de la fiction, absent de la tragédie racinienne, et fournissant à cet égard le contenu ironique, double celui de la chambre dont l'espace peut être rattaché à l'espace tragique racinien. La chambre, « reste de l'antre mythique (...), lieu invisible et redoutable où la puissance est tapie : chambre de Néron, palais d'Assuérus, Saints des Saints où loge le dieu juif », selon Barthes ${ }^{26}$, fait mesurer la portée de l'intrusion racinienne dans le discours proustien et évaluer la distance ironique sans que l'on puisse éliminer la présence effective de Proust dans sa chambre de liège. C'est la Chambre royale ramenée à sa dimension de lieu de sommeil, de repli aussi, qui permet dans le cas qui nous occupe d'adjoindre à la voix racinienne celle du héros de la Recherche comme celle du narrateur, doublée de celle de l'épistolier.

Le motif de l'identification à Assuérus court dans $\mathrm{La}$ Prisonnière tout autant que la relation avec Albertine s'inscrit dans l'espace intérieur de la chambre du héros dont Albertine n'a pas le libre accès. Françoise sert de messagère, et dès qu'elle en est avertie, Albertine entre : « Françoise m'a assuré que vous étiez éveillé et que je ne vous dérangerais $\operatorname{pas}^{27}$ ». Ses craintes sont identiques à celles de la mère du

25 Voir notre article "Une poétique de la disconvenance: burlesque et héroïcomique chez Proust ", in Poétiques du burlesque, Actes du colloque international du CRLMC de l'Université Blaise Pascal, 1996, édité par Dominique Bertrand, Champion, 1998, pp. 480487.

26 Sur Racine, Seuil, 1963, Points Essais, pp. 9-10.

${ }^{27}$ RTP, III, p. 626. 
narrateur du Contre Sainte-Beuve, elles s'expriment à travers le même emprunt à Racine, engendrent le même dialogue mais dont les variantes permettent de déceler derrière la voix citante, d'autres voix :

(...) la plus grande peur d'Albertine était d'entrer chez moi quand je sommeillais : «J'espère que je n'ai pas eu tort, ajouta-t-elle. Je craignais que nous ne me disiez: "Quel mortel insolent vient chercher le trépas ? Et elle rit de ce rire qui me troublait tant. Je lui répondis sur le même ton de plaisanterie : «Est-ce pour vous qu'est fait un ordre si sévère ? »Et de peur qu'elle l'enfreignit jamais j'ajoutai : «Quoique je serais furieux que nous me réveilliez.(...) Et pour adoucir j'ajoutai, en continuant de jouer avec elle la scène d'Esther (...) : « Je ne trouve qu'en vous je ne sais quelle grâce / Qui me charme toujours et jamais ne me lasse ${ }^{28}$ " (et à part moi je pensais : « $\mathrm{Si}$, elle me lasse souvent »)29.

Les termes sont à peu près les mêmes que ceux employés dans le Contre-Sainte Beuve, et permettent la confusion des voix, à laquelle on pourrait sans trop s'aventurer ajouter la fusion de la voix maternelle avec celle d'Albertine. Mais les commentaires du héros apportent la dissonance à ce qui était un duo sans fausse note dans la «Conversation avec Maman ». Albertine en ce cas, et fût-ce momentanément, rejoint le camp des fâcheux que Proust dans ses lettres rappelle à l'ordre en se réfugiant dans le personnage d'Assuérus.

La voix du héros amoureux se fait toutefois entendre par l'adjonction de vers réservés au roman, célébrant la grâce d'Albertine; la mention du jeu théâtral toutefois ramène à l'illusion la relation entre les deux personnages. Les vers cités, que l'on ne trouve nulle part ailleurs dans les écrits proustiens, consacrent à la fois l'exclusivité de la fiction et les mensonges et les masques qu'elle suggère. La voix citante en ce cas s'élève en solo pourrait-on dire, au-dessus des autres, dans la mesure où l'on n'en peut entendre aucune autre derrière elle, étant du domaine exclusivement romanesque.

\footnotetext{
${ }^{28}$ Esther, Acte II, Scène VII, vers 669-670.

${ }^{29}$ RTP, III, p. 627.
} 
Dialogue et dialogisme en ce cas s'installent au cœur du texte qui accueille la parole du narrateur, celle du héros, celle de l'héroïne confondue avec l'héroïne de la tragédie, sans que les notions de la théâtralité soient abandonnées.

(...) je me rappelai qu'Albertine m'avait dit une fois combien elle me trouvait l'air terrible quand j'étais en colère, et m'avait appliqué les vers d'Esther: «Jugez combien ce front irrité contre moi / Dans mon âme troublée a dû jeter d'émoi .../ Hélas! sans frissonner quel cœur audacieux / Soutiendrait les éclairs qui partent de vos yeux ${ }^{30}$ ? »

Si l'on se réfère au contexte immédiat de ces vers, c'est la parole du romancier et du créateur qui prend le dessus. «L'auguste majesté » d'Assuérus, le « trône sacré qu'environne la foudre », confèrent à la situation romanesque une ironie, démontée d'ailleurs par la parole de Proust épistolier lorsqu'il fait remarquer à son destinataire que sa seule ressemblance avec Assuérus est qu'on n'entre pas chez lui sans en être prié31, condition somme toute assez commune dans le cadre de la politesse mondaine. C'est bien le travail du créateur, par le regard oblique qu'il porte sur ses créatures, qui en augmentant la portée de la référence, crée l'ironie. Subtilement, celle-ci vise son héros et son héroïne, mais n'épargne pas le narrateur, qui tout en disant je continue d'obéir à l'auteur ${ }^{32}$ qui le dirige.

C'est quelques pages avant la fin de La Prisonnière et, par là même, peu de temps avant le départ d'Albertine, que l'on peut particulièrement observer, avec la conclusion du motif racinien, les effets ironiques qu'il engendre. Le héros, depuis son lit, rêve d'escapade avec une femme inconnue et se réjouit des bruits et des parfums de la matinée de printemps qui s'annonce :

${ }^{30} R T P$, III, p. 896 ; Esther, acte II, scène VII, vers 647-648 et 651 652.

${ }^{31}$ Voir supra, note 20.

32 Au sens où celui-ci détient l'autorité sur l'écriture et la conduite du récit. 
Odeur qu'accompagnait à tout moment l'appel des trompes d'automobiles qui passaient, sur lequel j'adaptais des paroles comme sur une sonnerie militaire : "Parisien, lève-toi, lève-toi, viens déjeuner à la campagne et faire du canot sur la rivière, à l'ombre sous les arbres, avec une belle fille, lève-toi, lève-toi. " Et toutes ces rêveries m'étaient si agréables que je me félicitais de la « sévère loi » qui faisait que, tant que je n'aurais pas appelé, aucun "timide mortel », fût-ce Françoise, fût-ce Albertine, ne s'aviserait de venir me troubler "au fond de ce palais » où "une majesté terrible / Affecte à mes sujets de me rendre invisible. ${ }^{33}$ »

Le mode d'insertion du texte racinien et le voisinage maintenu avec deux autres types de discours organisent dans le texte des jeux polyphoniques qui font le lien entre l'intertextualité et l'énonciation. L'implicite du « lève-toi soldat » que procure la mention de la «sonnerie militaire » assimilée probablement par le héros à «l'appel des trompes des automobiles ", le désir d'évasion résolu dans la rêverie, et l'introduction progressive du langage de la tragédie qui s'achève sur la vision grandiose et terrible d'Assuérus invisible au fond de son palais, ne manquent pas de créer un énoncé dans lequel l'énonciateur, ici le narrateur, place son personnage en position de décalage, aussi bien par son propre discours (fût-il le fruit de la rêverie) que par l'emprunt aux vers tragiques : la distorsion occasionnée par le mélange des temps, et par la transposition du pronom personnel, l'accentue. Le vers cité est en effet au présent, et installe le personnage qui parle à la première personne, dans une sorte de pérennité royale qui le met en marge du narrateur malgré la communauté du "je » inhérent au récit proustien. La parole ne s'en trouve pas moins dédoublée, au double titre de l'inadéquation du discours à la situation, introduite infailliblement par l'intertextualité classique, et de la disjonction des voix narratives qui laissent la place à l'écrivainn ${ }^{34}$.

${ }^{33}$ RTP, III, p. 913 ; Esther, Acte I, Scène III, voir supra p. 5, note 14.

34 Marcel Muller, dans Les voix narratives dans « La Recherche du temps perdu ", op. cit., p. 98-100, y voit un lien avec ce qu'il appelle 
On pense à ce «personnage qui dit je (et qui n'est pas toujours moi) » évoqué par Proust à propos de son œuvre, et que nous citions plus haut.

La conception polyphonique de l'ironie ${ }^{35}$ entre en rapport étroit avec la pratique de la citation, et particulièrement de la citation classique ; celle-ci engendre des situations de décalage qui en ouvrant sur des notions de parodie et de travestissement, compromettent immanquablement l'unicité de la voix narrative et contribuent de surcroît à donner cette tonalité particulière qui constitue l'un des aspects du comique proustien.

la «mise en congé du héros " qui correspond à une expérience contemplative favorable en général au travail de l'écrivain.

35 Elle met en jeu des distorsions entre locuteur et énonciateur, voir Oswald Ducrot, Le Dire et le dit, Editions de Minuit, 1984, pp. 208214. 